\title{
Multiple solutions of asymmetric potential bistable energy harvesters: numerical simulation and experimental validation
}

Wei Wang ${ }^{1}$, Junyi Cao ${ }^{1, a}$, Chris R. Bowen ${ }^{2}$, Grzegorz Litak ${ }^{3,4}$

${ }^{1}$ Key Laboratory of Education Ministry for Modern Design and Rotor-Bearing System, School of Mechanical Engineering, Xi'an Jiaotong University, Xi'an, 710049, China

${ }^{2}$ Materials and Structures Centre, Department of Mechanical Engineering, University of Bath, Bath, BA27AY, UK

${ }^{3}$ Faculty of Mechanical Engineering, Lublin University of Technology, Nadbystrzycka 36, Lublin 20-618, Poland

${ }^{4}$ Department of Process Control, AGH University of Science and Technology, Mickiewicza 30, 30-059 Krakow, Poland

\begin{abstract}
In this paper we investigate the multiple solutions of nonlinear asymmetric potential bistable energy harvesters (BEHs) under harmonic excitations. Basins of attraction under certain excitations explain the existance of multiple solutions in the asymmetric potential BEHs and indicate that the asymmetric system has a higher probability to oscillate in the deeper potential well under low and moderate excitation levels. Thus, the appearance of asymmetric potentials in BEHs has a negative influence on the output performance. Average output powers under different excitation frequencies and initial conditions illustrate that the asymmetric potential BEHs are more likely to achieve high-energy branch (HEB) with initial conditions in the shallower potential well, and the probability is influenced by the degree of asymmetry of the BEHs. Finally, experiments are carried out, and results under constant and sweep frequency excitations demonstrate that the output performance will be actually improved for the asymmetric potential BEHs if the initial oscillations a e-mail:caojy@mail.xjtu.edu.cn


are from the shallower potential well.

Keywords: Energy harvesting; Bistable; Asymmetric potential; Multiple solutions. 


\section{Introduction}

In recent decades, the rapid development of low-power consumption devices such as miniaturized sensors and wearable electronics has motivated the emerging trend in small-scale energy supply technologies[1]. Piezoelectric energy harvesting from ambient vibrations is a promising method to supply electricity for these low-power devices to reduce reliance on batteries and extend their lifespan[2-4]. In this field, linear energy harvesters (LEH) with various structures have been previously investigated extensively[5]. However, the sensitivity of LEHs to a single resonance frequency has limited their wide scale application[6].

To remedy this problem, a variety of nonlinear phenomena have been introduced to piezoelectric energy harvesting devices to broaden their effective frequency range of operation[7,8]. For instance, Stanton et al.[9] reported a monostable energy harvester by tuning nonlinear magnetic interactions around the end mass. Zhang et al.[10] presented a piezoelectric harvester based on the nonlinear energy sink, which could simultaneously achieve broadband energy harvesting and vibration suppression. Further, Fan et al.[11] explored a monostable structure by introducing symmetric magnetic attraction to a piezoelectric cantilever beam and two stoppers to confine the maximum deflection for performance enhancement. Due to the sensitivity to excitations, bistable systems have attracted lots of attention from many researchers. Cottone et al.[12] proposed a bistable inverted pendulum for piezoelectric energy harvesting. Erturk et al.[13,14] introduced a bistable piezomagnetoelastic structure, and investigated into its high-energy branches (HEB) over a range of excitation frequencies. Additionally, Litak et al.[15] numerically simulated the response of a bistable energy harvester (BEH) under random base excitation and showed that the system exhibited a stochastic resonance phenomenon. Arrieta et al.[16] considered a cantilevered piezoelectric bistable 
composite for broadband energy harvesting and revealed its wide bands of power conversion. Masana et al.[17] evaluated an axially loaded BEH subjected to band-limited noise and concluded that the $\mathrm{BEH}$ outperformed the monostable design for all acceleration levels. More recently, Wolszczak et al.[18] considered a BEH with additional impacts. To further improve the performance of harvesters under lower excitation levels, tristable configurations sensitive to vibrations were reported by Kim and Seok[19], Zhou et al.[20], Cao et al.[21] and Li et al.[22].

Based on the multistable configurations, many researchers focused on the investigation into their nonlinear dynamic characteristics by a variety of approaches. Masana and Daqaq[23] exploited the super-harmonic frequency bands of a BEH, indicating that it could excite large-amplitude inter-well motions at fraction integers of the fundamental frequency. Cao et al.[24] investigated the dynamics of a fractionally damped $\mathrm{BEH}$ and concluded that the harvester possessed a usable frequency bandwidth over a wide range of low-frequency excitations. Harris et al.[25,26] examined the multiple vibration patterns of BEHs under different excitation conditions by the method of wavelet, phase portraits, multiscale entropy, phase space reconstruction and "0-1" test. In particular, Syta et al.[27] numerically considered the multiple solutions and global dynamical properties of a $\mathrm{BEH}$ under same excitation condition by the basins of attraction. They also investigated the multiple solutions of tristable energy harvesters with different geometries of the potential and initial conditions[28]. However, the above investigations into the multiple solutions of multistable harvesters are still limited to numerical simulations.

Another issue to be noted is that the above investigations into multistable harvesters have always concentrated on systems with symmetric potential functions. However, it is challenging or almost impossible to obtain a completely symmetric potential function during the fabrication of a 
multistable configuration due to imperfections in structural and material properties. Badzey et al.[29] reported the stochastic resonance in bistable nanomechanical oscillators and observed an asymmetric potential induced by the change of temperature. Also, some researchers indicated that the asymmetry of the potential in bistable systems can weaken the phenomenon of stochastic resonance[30-32]. While under the excitation of correlated multiplicative and additive noises, it was demonstrated that the effect of potential asymmetry on signal-to-noise ratio strongly depends on the values of noise intensity and cross-correlation strength between noises[33]. In addition, the asymmetric potential issue has attracted attention from researchers devoted to the investigation of vibrational resonance[34]. In the field of energy harvesting, He and Daqaq[35] considered an asymmetric quadratic nonlinearity similar to the one proposed by Halvorsen[36] and demonstrated that it appeared to degrade the performance for low to moderate white noise intensity. Zhou et al.[37] theoretically investigated the responses of asymmetric tristable harvesters by deducing the harmonic balance solutions, which were validated by basins of attraction. However, the experimental characterization of multistable harvesters with different initial conditions remains an open issue, especially the terms of strategies for the performance enhancement of the asymmetric potential BEHs.

Therefore in this paper, numerical and experimental investigations into the multiple solutions of asymmetric potential BEHs are carried out. Results indicate that the initial conditions and asymmetric degrees have a significant influence on the output response of the asymmetric system, where initially oscillating the harvester from the shallower potential well will achieve HEB to generate a large power output. The remainder of the paper is organized as follows. In Sect. 2, we present the electromechanical model of the asymmetric potential BEH studied in this paper. 
Numerical investigations into the influence of initial conditions and asymmetric degrees on the multiple solutions and output performance of the system are undertaken in Sect. 3. In Sect. 4, experimental investigations of the multiple responses of the asymmetric BEH under constant and sweep frequency excitations are carried out and the conclusion is given in Sect. 5.

\section{Configuration and electromechanical model}

Figure 1 illustrates the schematic of the $\mathrm{BEH}$ investigated in this paper. It is composed of a stainless steel substrate, two lead zirconate titanate (PZT) piezoelectric layers at the root, tip magnet attachments and external magnets. By modulating the appropriate system parameters of $d, h, \vartheta$, a bistable configuration could be obtained. Due to the imperfections in the structures and materials, the manufactured bistable system will have an asymmetric potential energy function[38,39].

The electromechanical model of the asymmetric potential BEH is expressed as

$$
\left\{\begin{array}{l}
m r^{\prime \prime}(\bar{t})+c r^{\prime}(\bar{t})-k_{1} r+k_{2} r^{2}+k_{3} r^{3}-\theta \bar{v}(\bar{t})=\bar{F} \cos (\bar{\Omega} \bar{t}) \\
C_{\mathrm{p}} \bar{v}^{\prime}(\bar{t})+\bar{v}(\bar{t}) / R+\theta r^{\prime}(\bar{t})=0
\end{array}\right.
$$

where $m, c$ and $\theta$ are respectively the equivalent mass, damping and electromechanical coupling coefficient. $C_{\mathrm{p}}$ is the capacitance of the PZT layers and $R$ is the load resistance. $r$ and $\bar{v}$ are the tip displacement of the harvester and the output voltage, respectively. $\bar{F}$ is the amplitude of external excitation force. $k_{1}, k_{2}$ and $k_{3}$ are respectively the coefficients of the nonlinear terms characterizing the nonlinear restoring force. Further, ()$^{\prime}$ denotes a derivate with respect to time $\bar{t}$. 


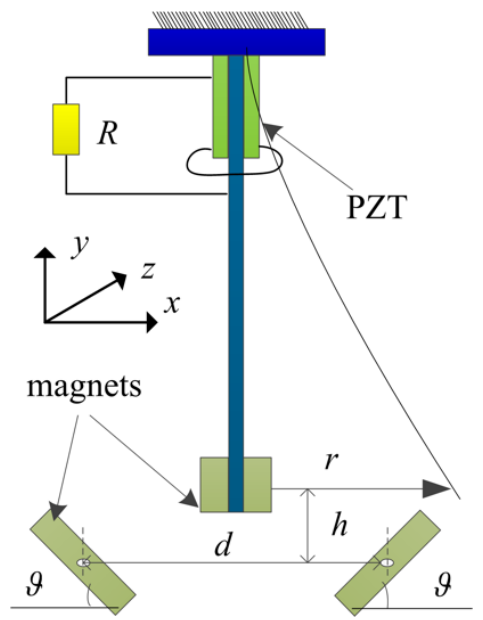

Fig. 1. Schematic of the bistable energy harvester $(\mathrm{BEH})$, showing magnets, piezoelectric lead zirconate titanate (PZT) attached to cantilever and load resistance $(R)$.

For convenience, the equations of motion can be further nondimensionalized as follows by introducing $x=r / l_{c}, t=\bar{t} \omega_{n}$ and $v=C_{p} \bar{v} / \theta l_{c}$ :

$$
\left\{\begin{array}{l}
2 \xi x+\beta x^{2}+\delta x^{3}-\kappa^{2} v=F \cos (\omega t) \\
\alpha v+x=0
\end{array}\right.
$$

where $l_{\mathrm{c}}$ is a length scale and $\omega_{n}=\sqrt{k_{1} / m}$ is the natural frequency. Then, $x, v$ and $t$ are the dimensionless displacement, voltage and time respectively, $\xi$ is the damping ratio, $\kappa^{2}$ is the dimensionless electromechanical coupling coefficient and $\alpha$ is the ratio between the period of the mechanical system and the time constant of the harvesting circuit. $\beta$ and $\delta$ are the coefficient of quadratic and cubic nonlinearity. Further, $F$ and $\omega$ is the dimensionless excitation force and frequency and the dot above the parameters indicates a derivate with respect to dimensionless time $t$.

For the dimensionless model, the average output power of the system is given by

$$
P=E\left[\alpha \times v^{2}(t)\right]
$$

\section{Numerical simulation}

In this section, numerical simulations of the asymmetric potential BEHs will be undertaken to evaluate the influence of initial conditions and the degree of asymmetry of the potential function on 
the output response of the system. Basins of attraction and power outputs are initially applied to illustrate the sensitivity of the asymmetric potential BEH to initial conditions. The degree of asymmetry of the system is then studied based on the power outputs of the harvester. All the numerical simulations are carried out by using the four-order Runge-Kutta method. Furthermore, three asymmetric potential BEHs are considered in the following simulations with $\beta$ equaling to 0.15 , 0.25 , and 0.35 , and the corresponding potential energy functions are shown in Fig. 2, along with the symmetric potential energy function for comparison. It is seen that the potential well on the left becomes much deeper, while the right one becomes shallower with the increase in the value of $\beta$.

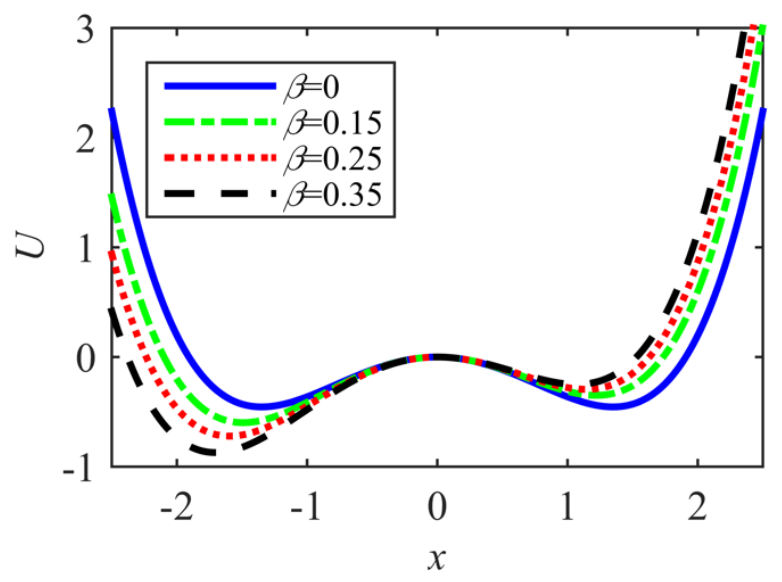

Fig. 2. Potential energy functions when $\beta$ equals to $0,0.15,0.25$, and 0.35 .

\subsection{Influence of initial condition}



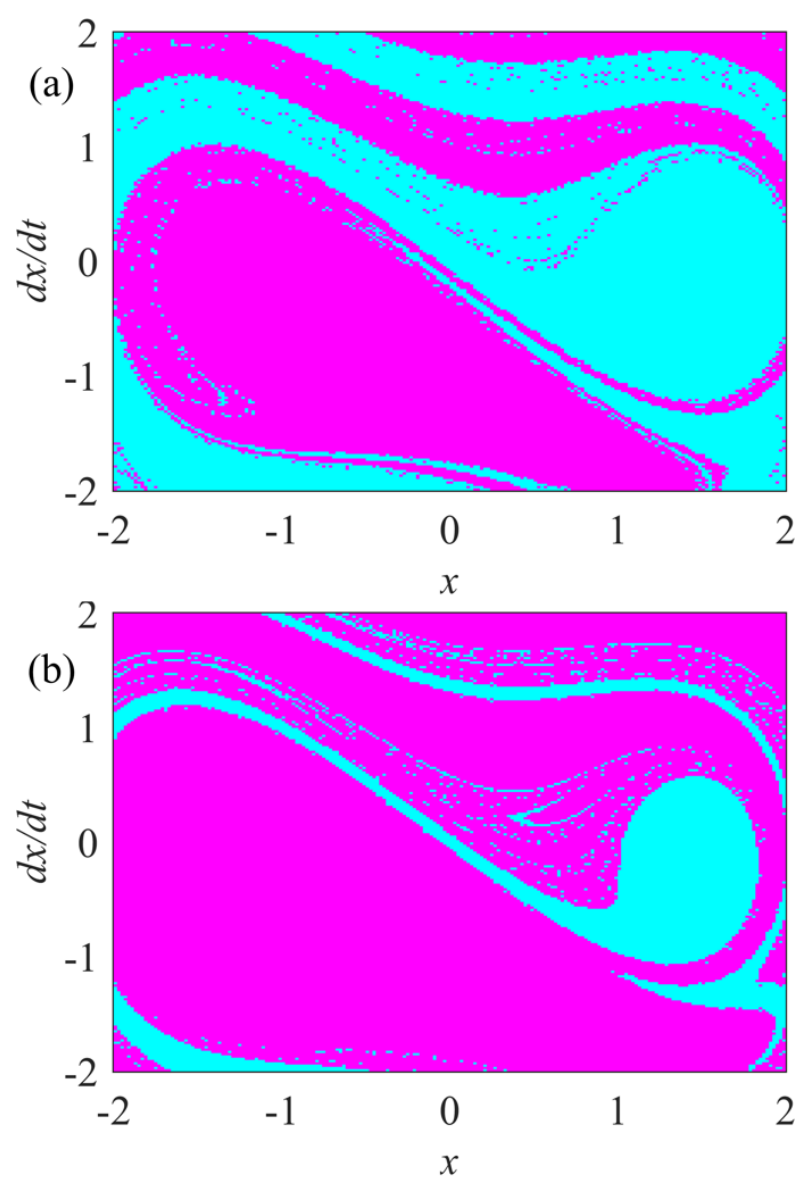

Fig. 3. Basins of attraction for BEH under an excitation of $F=0.2$ and $\omega=1$ : (a) $\beta=0$; (b) $\beta=0.15$.

For a completely symmetric potential BEH with $\beta=0$, the basin of attraction under an excitation of a relatively small level, $F=0.2$, and $\omega=1$, is illustrated in Fig. 3a. For the basins of attraction in this paper, each simulation is performed for 300 cycles to identify the final state of the system. In the figure, the pink area represents the oscillator with a final state in the left potential well, while cyan area for those in the right one. If there is insufficient energy for the BEH to travel across the potential wells, the oscillator has almost the same probability to vibrate in each well directly or after a transient chaos condition[40]. This indicates that the initial conditions has no influence on the power output for a symmetric potential $\mathrm{BEH}$ under low excitations. However, the result will be completely different when an asymmetric potential is introduced to the $\mathrm{BEH}$ for the reason that different depths of potential wells appear. Figure $3 \mathrm{~b}$ shows the basin of attraction of an asymmetric 
potential BEH with $\beta=0.15$ under the same excitation. A higher probability of $82.23 \%$ is indicated for the asymmetric potential BEH to vibrate with a final state in the deeper potential well (pink) and only $17.77 \%$ in the shallower one. A different final state of the oscillator in the asymmetric potential $\mathrm{BEH}$ will result in different power outputs. Hence, the asymmetric potential energy function of a $\mathrm{BEH}$ has an influence on the power output performance. In this case, the relatively small excitation level leads to small orbits (single well) solutions. Note that the border between the two basins is eroded (forming a fractal layer). This could indicate possibilities of appearance of chaotic transients or even stationary chaotic solutions for larger amplitude responses. However, for higher excitation levels we expect large orbits with passing through the potential barrier.
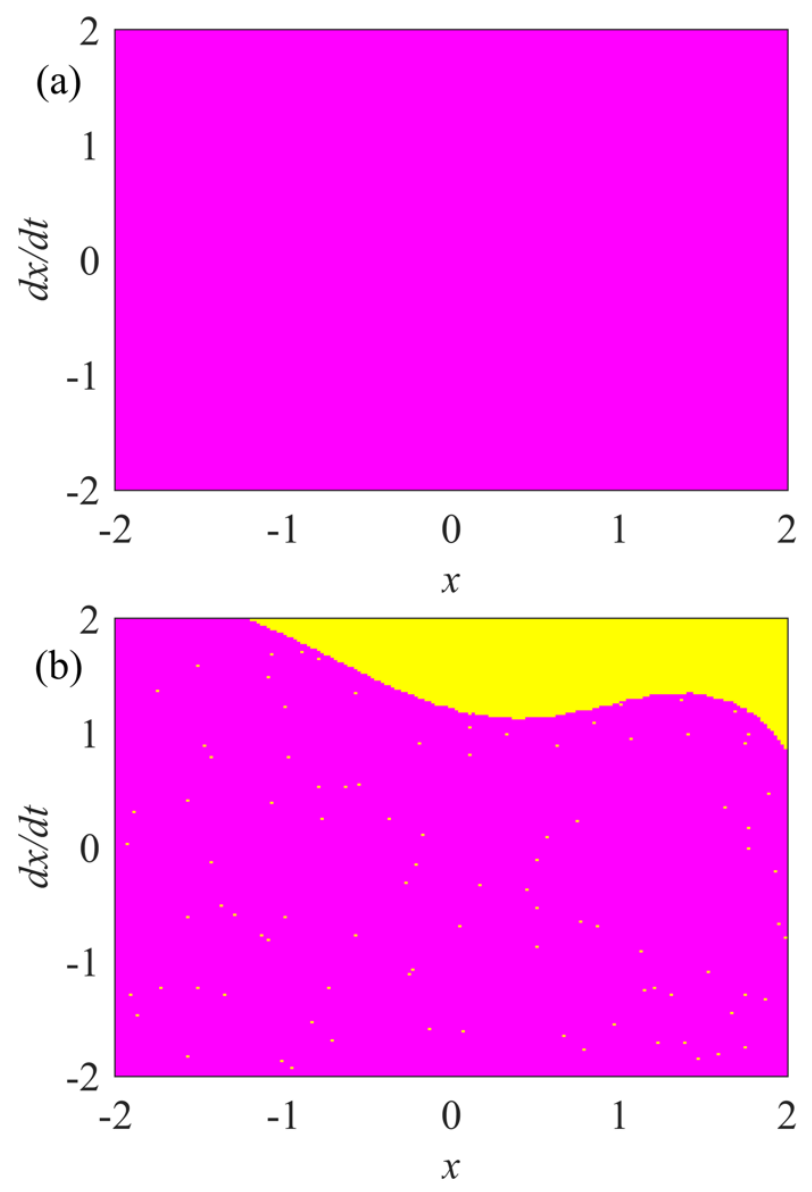

Fig. 4. Basins of attraction for BEH with $\beta=0.15$ under an excitation of $\omega=1$ : (a) $F=0.3$; (b) $F=0.45$. 
With the same coefficient of quadratic nonlinearity, the influence of excitation level on the final state of the BEH is investigated. Figure 4a shows the basin of attraction of the asymmetric potential $\mathrm{BEH}$ under an excitation of $F=0.3$ and $\omega=1$. Under this excitation level, the oscillator in $\mathrm{BEH}$ has sufficient energy to travel across the shallower potential well, while it has insufficient energy to cross the deeper one. Thus, the oscillator has a probability of $100 \%$ to have a final state in the deeper potential well, indicated as pink in Fig. 4a. With an increase of excitation level to $F=0.45$, see Fig. $4 b$, the oscillator could travel across the potential barrier from both the shallower and deeper potential wells. Nevertheless, the probability to cross the potential wells and achieve HEB (yellow area, which represents inter-well oscillation) is only $13.63 \%$, which is small in comparison to $86.37 \%$ with a final state in the deeper potential well (pink). Therefore, it can be concluded that the appearance of asymmetric potential energy functions in BEHs will result in multiple solutions characterizing different power outputs and have a negative effect on the performance.

In addition, the influence of asymmetric potential function on the output performance of the BEH under different excitation levels and frequencies are studied through the power outputs of the harvester. The power outputs are calculated through the last 100 cycles of each simulation performed for 300 cycles. Since the BEH always oscillates from one of the potential wells in experiments, we calculate here the power outputs of the BEH vibrating from two potential wells with various initial speeds to compare the performance. Under the excitation level of 0.4 and frequency of $0.7,0.8,0.9$, 1.0 and 1.1, the dimensionless output powers of the asymmetric potential $\mathrm{BEH}(\beta=0.15)$ oscillating from shallower potential well with various initial speeds ranging from -2 to 2 are described in Fig. 5a. When the excitation frequency is 0.7 , oscillating with different initial speeds result in different output performance. The probability to vibrate on HEB with a large output power of 0.51 is $51.87 \%$, while 
$48.13 \%$ for oscillating on low-energy branch (LEB) with a lower power of 0.025 generated. For comparison, the oscillations on HEB and LEB are illustrated in Fig. 6a and 6b, respectively. The phase orbits and Poincare maps of the system clearly indicate the vibration of the system on different orbits. As the frequency increases to 0.8 , the BEH oscillating with various initial speeds has a larger probability of $68.83 \%$ to generate a larger output power of 0.60 on HEB, and the output power will continually increase to 0.69 with a smaller probability of $22.19 \%$ when the excitation frequency is 0.9. Except for two scattered points, the oscillation of the asymmetric potential BEH is on LEB when the frequency is increased to 1.0. Furthermore, the chaotic oscillations, indicated in Fig. 6c, are obtained for all initial speeds and the power output is approximately 0.25 when the excitation frequency is 1.1 .
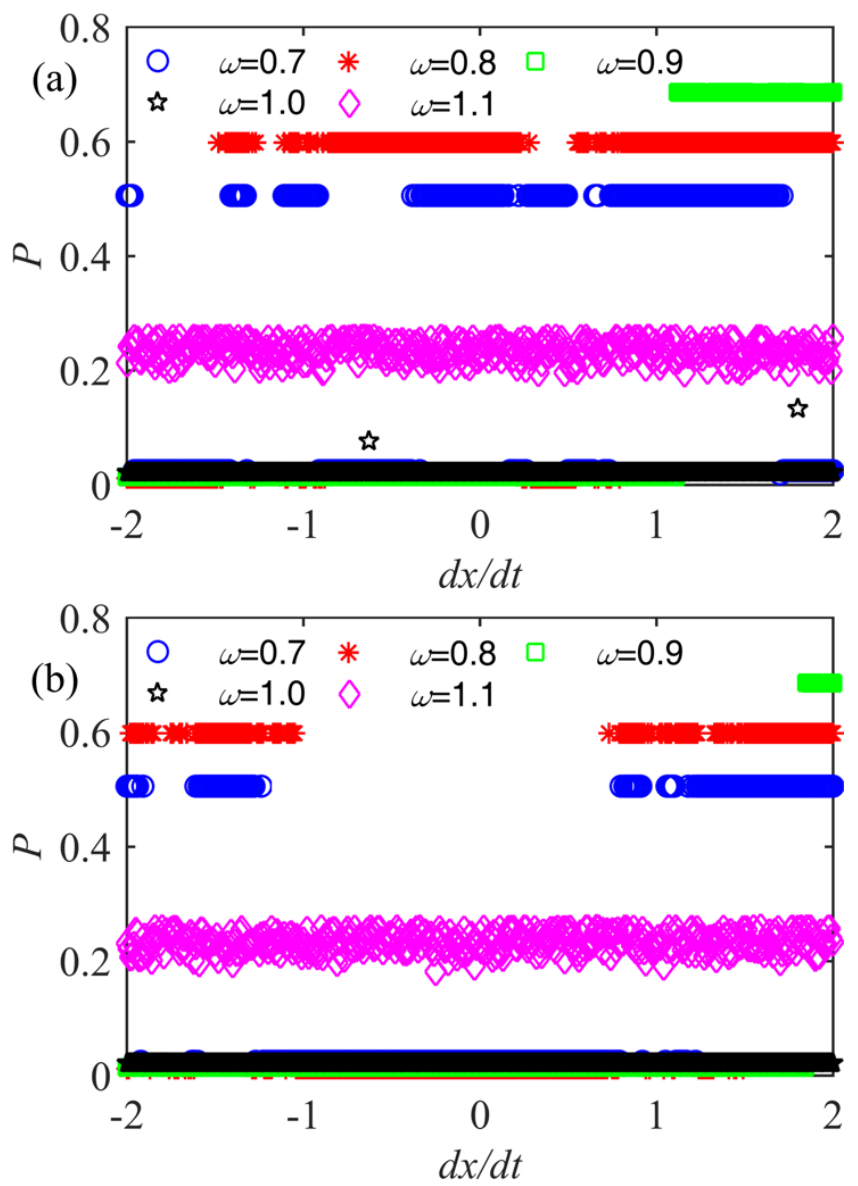

Fig. 5. Average output powers of $\mathrm{BEH}$ with $\beta=0.15$ under excitation level of 0.4 and different 
frequencies when oscillating from the (a) shallower and (b) deeper potential wells with various initial speeds.

With the same excitation conditions, the output powers of the asymmetric potential BEH oscillating from the deeper potential well with various initial speeds are plotted in Fig. 5b. The probability to achieve HEB under excitation frequencies of $0.7,0.8$ and 0.9 are respectively $34.41 \%$, $40.65 \%$ and $3.99 \%$, which are relatively low compared to that oscillating from the shallower potential well. When the frequency is 1.0 , the vibration of the BEH is always on LEB. And the chaotic motions under excitation of 1.1 are not influenced by the initial conditions. Another important point to be noted is that the initial speeds resulting in HEB when oscillating from deeper potential well are overall larger than that oscillating from shallower potential well. In other words, it is more likely to achieve HEB and generate a large power output with an initial condition in the shallower potential well.
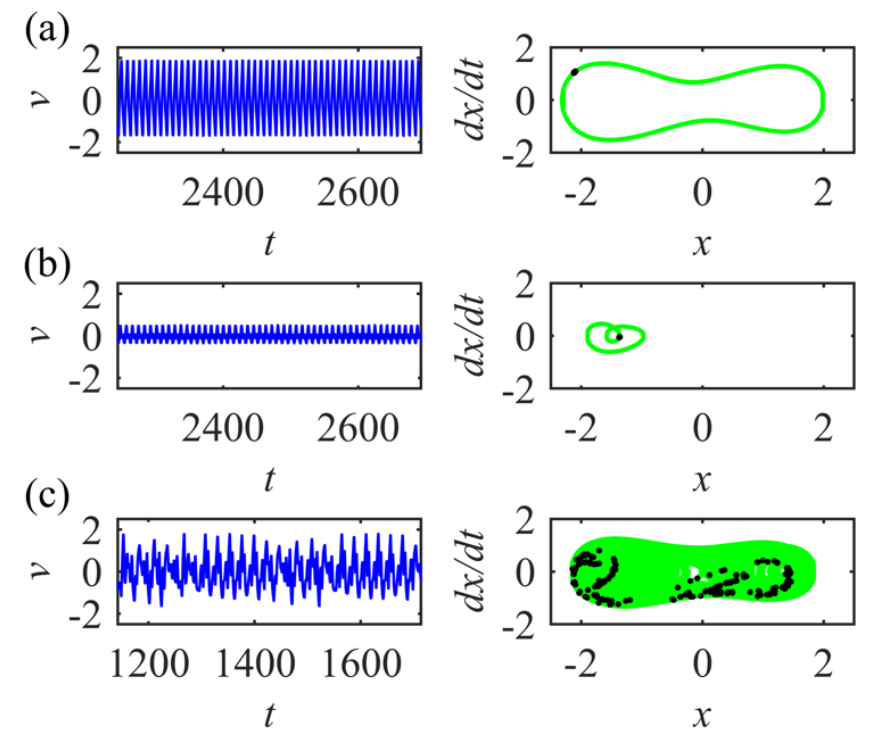

Fig. 6. Voltage response, phase orbit and Poincare map of BEH with $\beta=0.15$ when oscillating from the shalllow potential well under excitation of $F=0.4$ with different initial speed: (a) $\omega=0.7$, $\not \& 0)=0$; (b) $\omega=0.7, \quad \&(0)=-0.5$; (c) $\omega=1.1, \quad \not \& 0)=0$. 

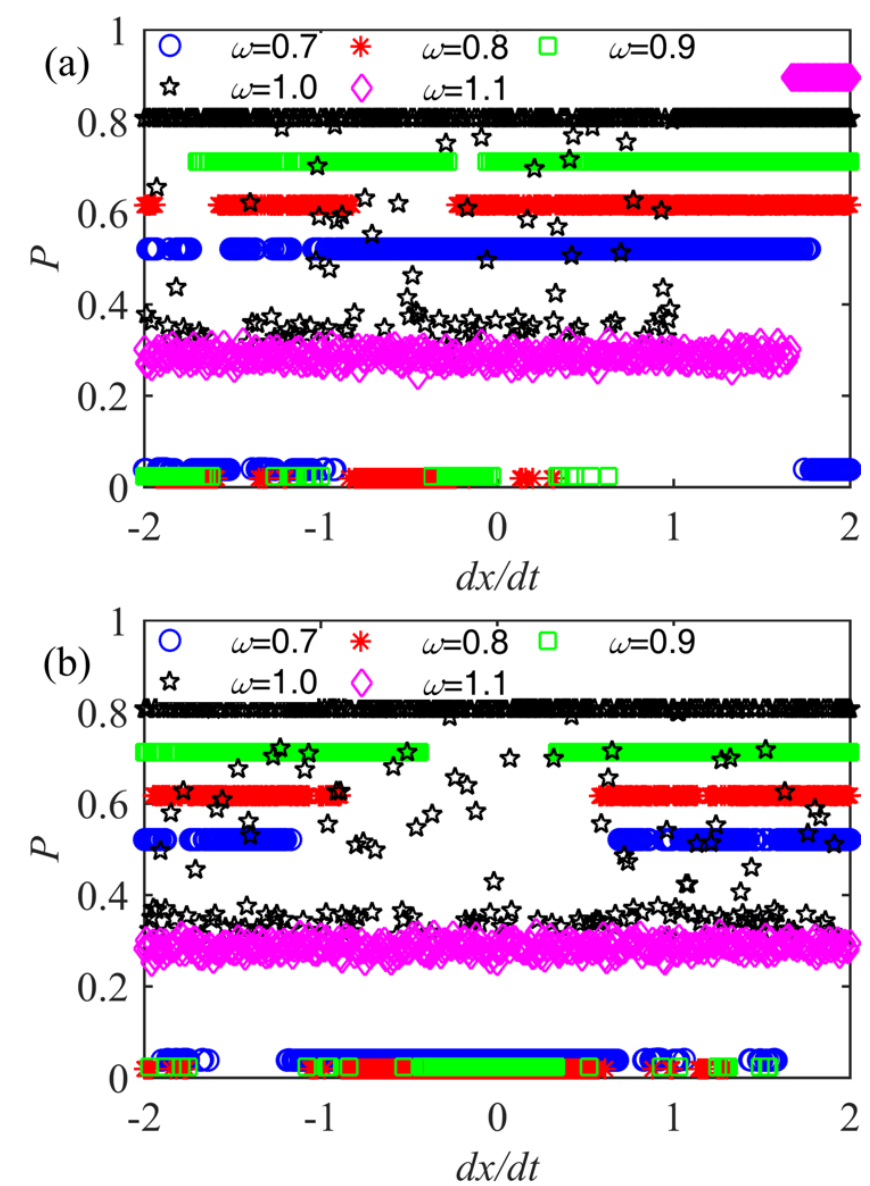

Fig. 7. Average output powers of BEH with $\beta=0.15$ under excitation level of 0.5 and different frequencies when oscillating from the (a) shallower and (b) deeper potential wells with various initial speeds.

When the excitation is increased to 0.5 , Figure $7 \mathrm{a}$ indicates the dimensionless output powers of the $\mathrm{BEH}(\beta=0.15)$ under different excitation frequencies oscillating from the shallower potential well with various initial speeds. For an excitation frequency of 0.7 , a large output power of 0.52 is obtained on HEB with a probability of $79.05 \%$. When the frequencies are 0.8 and 0.9 , larger output powers are achieved at 0.62 and 0.71 , corresponding to the probabilities of $73.57 \%$ and $82.04 \%$. At a frequency of 1.0, except for the scattered points indicating chaotic motion, the asymmetric potential $\mathrm{BEH}$ often vibrates on HEB (76.81\%) with a large output power of 0.81 generated. When the frequency is further increased to 1.1 , the asymmetric potential $\mathrm{BEH}$ has a probability of $8.48 \%$ to 
generate a large power output of 0.90 , while with a probability of $91.52 \%$ to exhibit chaotic motion. Compared with the results under excitation level of 0.4 , not only are the output powers improved, but also the probability to oscillate on HEB is increased due to larger excitation level. When oscillating from the deeper potential well, see Fig. $7 b$, the probability to generate large output powers are $42.89 \%, 56.11 \%, 75.56 \%$ and $63.84 \%$ under excitation with frequencies of $0.7,0.8,0.9$ and 1.0 , respectively. While for frequency of 1.1, oscillating from deeper potential well with all initial speeds always results in chaotic vibration. From the numerical simulation above, it is demonstrated that the asymmetric potential energy function in $\mathrm{BEH}$ has a negative impact on the performance of the system, while it is more likely to achieve HEB with a large output power by oscillating from the shallower potential well.

\subsection{Influence of asymmetric degree}

In practical conditions, it is difficult to control the degree of the asymmetry in a BEH. Therefore, the influence of degree of asymmetry on the output performance of the BEH will be investigated in this section by changing the value of $\beta$. When oscillating from the shallower potential well with various initial speeds, the output power of the asymmetric potential $\mathrm{BEH}$ with $\beta=0.25$ under excitation level of 0.5 and different frequencies are shown in Fig. 8a. For excitation frequencies of $0.7,0.8,0.9$ and 1.0, the larger output powers on HEB are $0.53,0.62,0.72$ and 0.81 with probabilities of $58.85 \%, 73.82 \%, 73.82 \%$ and $33.42 \%$, respectively. However, the lower probabilities of $33.42 \%, 46.88 \%, 52.12 \%$ and $4.24 \%$ are observed for the situations vibrating from the deeper potential well shown in Fig. 8b. As the excitation frequency is increased to 1.1, the vibrations of the asymmetric potential BEH are always chaotic when oscillating from deeper potential well, while it also has a very small probability of $8.48 \%$ to generate a larger output power of 0.90 when with an 
initial position in the shallower potential well.
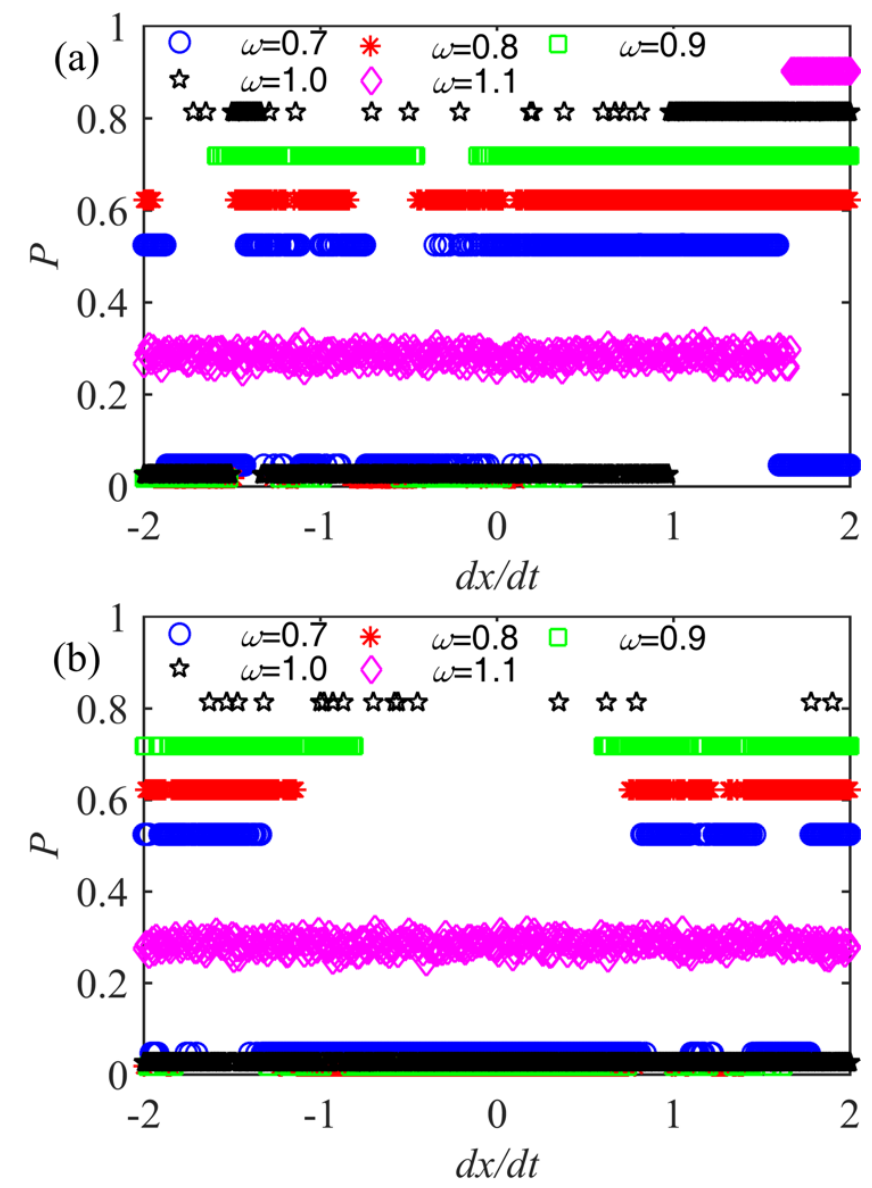

Fig. 8. Average output powers of BEH with $\beta=0.25$ under excitation level of 0.5 and different frequencies when oscillating from the (a) shallower and (b) deeper potential wells with various initial speeds.

With $\beta$ further increasing to 0.35 , the average power outputs of the BEH under excitation level of 0.5 and various initial speeds are illustrated in Fig. 9a and 9b for oscillating from the shallower and deeper potential wells, respectively. It is seen that the output power is only 0.02 on LEB under an excitation frequency of 0.7 for any initial conditions. When the excitation frequency is increased to 0.8 and 0.9 , large output powers of 0.63 and 0.72 on HEB are obtained with probabilities of $70.82 \%$ and $69.58 \%$ with initial displacement in the shallower potential well. The probabilities are $37.16 \%$ and $41.15 \%$, respectively, for those with initial position in deeper potential well. As 
excitation frequency further increases to 1.0 and 1.1 , the probability to achieve a larger output power on HEB decrease to $29.43 \%$ and $7.73 \%$ when vibrating from shallower potential well. Obviously, the oscillation from the deeper potential well are on LEB all the time expect for one scattered point with chaotic motion. By comparing the results to that in Sect 3.1, it is noted that an increase in the degree of asymmetry in the BEH not only has an effect on the output power but also significantly influence the probability to achieve HEB with large power outputs.
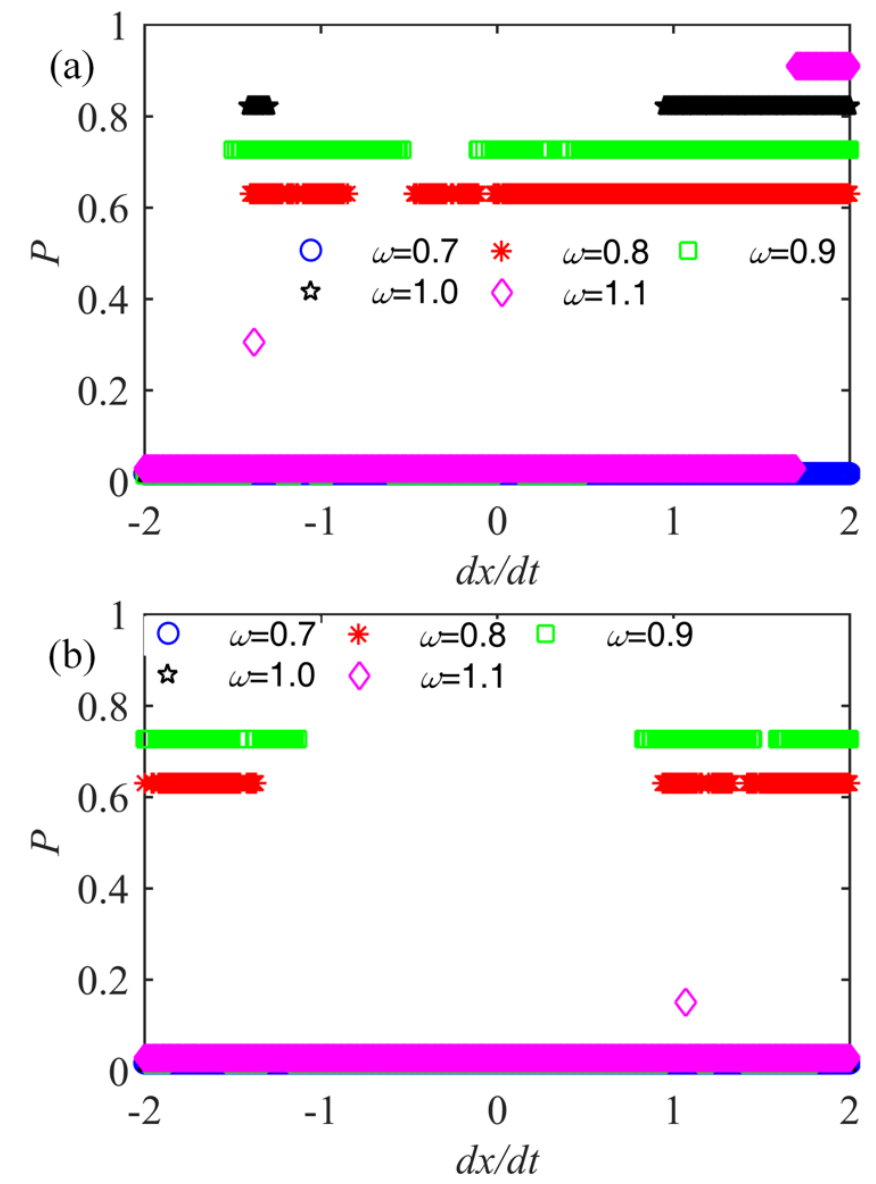

Fig. 9. Average output powers of $\mathrm{BEH}$ with $\beta=0.35$ under excitation level of 0.5 and different frequencies when oscillating from (a) shallower and (b) deeper potential wells with various initial speeds.

\section{Experimental results and analysis}

In this section, experiments are carried out to demonstrate the numerical simulations above. A 
$\mathrm{BEH}$ with an asymmetric potential energy function shown in Fig. 10 is designed and excited under constant and sweep frequency excitations by a vibration exciter (JZK-50) controlled by a signal generator (VT-9002-1) and a power amplifier (YE5874A). An acceleration sensor (EA-YD-185) is applied to measure the excitation acceleration and an oscilloscope (MSOX3052A) with probe resistance of $10 \mathrm{M} \Omega$ is to collect the experimental data.

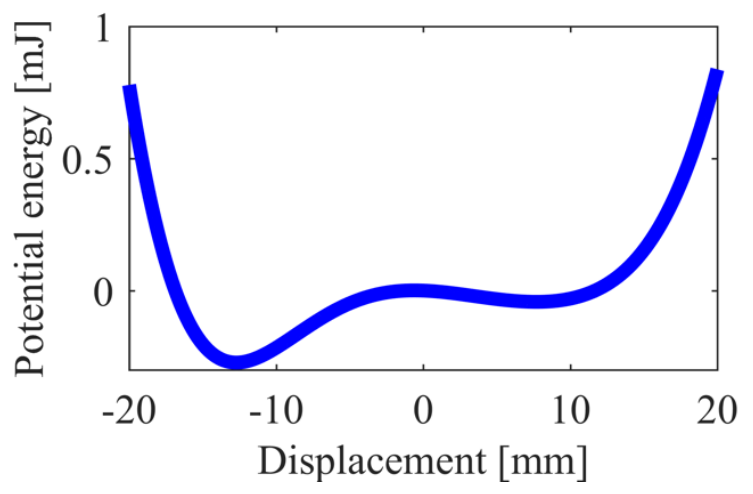

Fig. 10. Potential energy function of the asymmetric potential BEH in experiment.
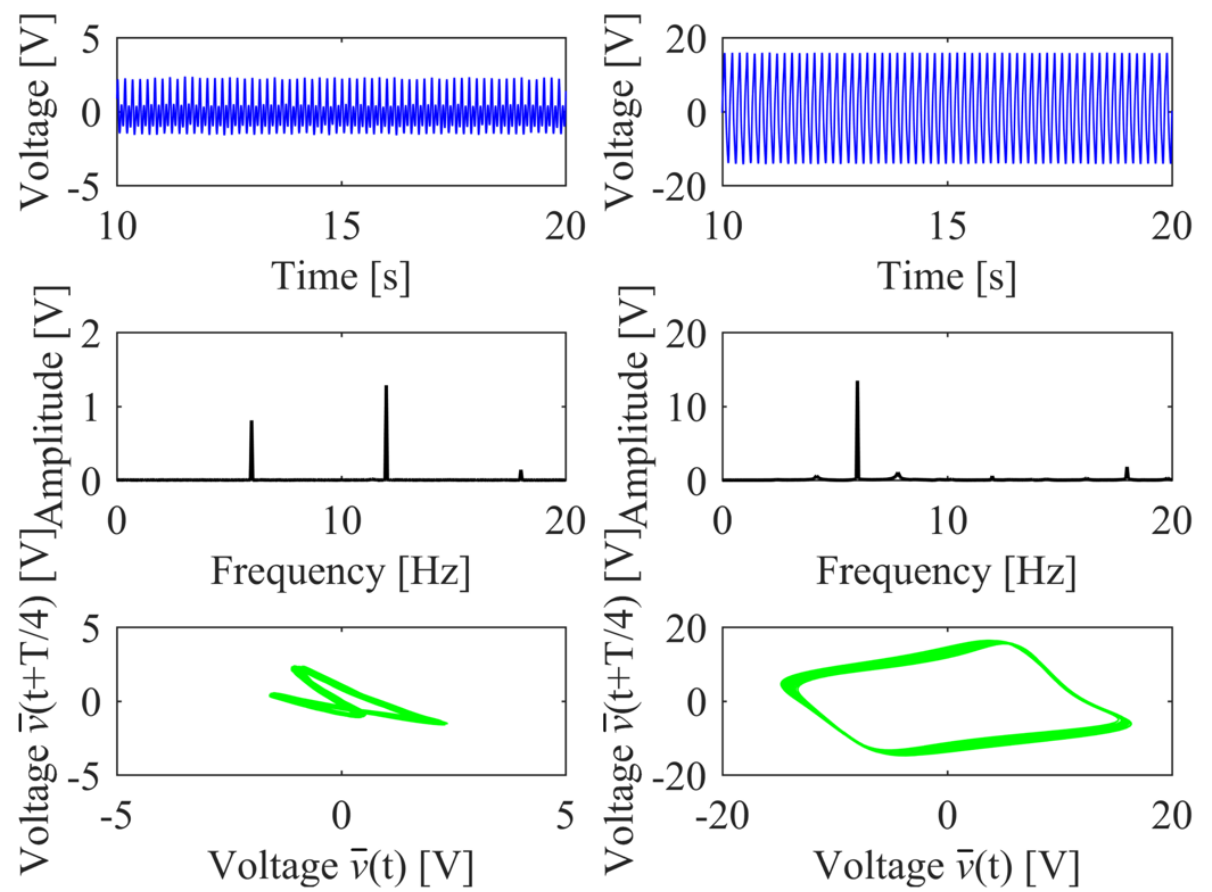

(a)

(b)

Fig. 11. Output voltage, frequency spectrum and reconstructed phase portrait of BEH under an excitation of $3 \mathrm{~m} / \mathrm{s}^{2}$ and $6 \mathrm{~Hz}$ with an initial position in the (a) deeper and (b) shallower potential 
well

Figure 11a shows the case of the asymmetric potential BEH oscillating from the deeper potential well under an excitation of $3 \mathrm{~m} / \mathrm{s}^{2}$ and $6 \mathrm{~Hz}$. Apart from the time series of the voltage output and corresponding frequency spectrum, we also plot the reconstructed phase portrait by using the delayed coordinate. The delay time $\mathrm{V} t$ has been chosen in the standard way using a quarter of the excitation period. Note that the maximum output voltage in this case is only $2.3 \mathrm{~V}$ when oscillating in single well. Through the frequency spectrum and reconstructed phase portrait, one can clearly identify the periodic multi-frequency response on LEB. Not only can the excitation frequency of $6 \mathrm{~Hz}$ be observed, but also a double frequency of $12 \mathrm{~Hz}$ can be seen as a main component. This is evidently an appearance of super-harmonic solution where the dominating frequency of the response is two times of the excitation frequency. These is a single well solution in the presence of an asymmetric potential, such solutions can be also found in a system with higher number of potential wells[41]. When with an initial position in shallower potential well, the voltage response, frequency spectrum and reconstructed phase portrait are illustrated in Fig. 11b. In this condition, the asymmetric potential $\mathrm{BEH}$ could travel across the potential well and generate a maximum voltage of $15.8 \mathrm{~V}$ on HEB. Unlike the results when the system is initially vibrating from the deeper potential well, the vibration of the $\mathrm{BEH}$ in this condition is periodic single frequency response with only the excitation frequency of $6 \mathrm{~Hz}$.

As the excitation frequency increases to $8 \mathrm{~Hz}$, the corresponding results with an initial position in the deeper potential well are shown in Fig. 12a. A maximum voltage of $1.4 \mathrm{~V}$ is generated on LEB and the vibration of $\mathrm{BEH}$ is indicated as a single frequency response with a main component at $8 \mathrm{~Hz}$. The experimental result shown in Fig. 12b for oscillating from shallower potential well is a different 
case compared to the results above. The voltage response is characterized as a chaotic vibration condition by the reconstructed phase portrait, which is consistent with the frequency spectrum indicating a more complex multi-frequency response. When the excitation frequency further increases to $9.5 \mathrm{~Hz}$, the voltage responses, frequency spectrums and reconstructed phase portraits with an initial position in the deeper and shallower wells are shown in Fig. 13a and 13b. Different initial conditions in the shallower and deeper potential wells result in the same chaotic response characteristics.
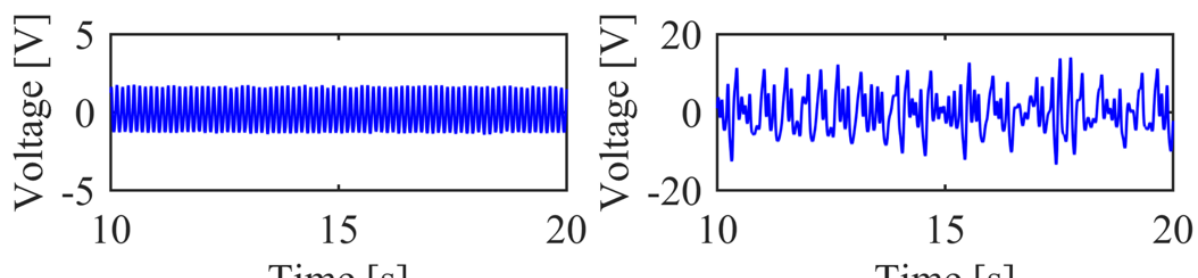

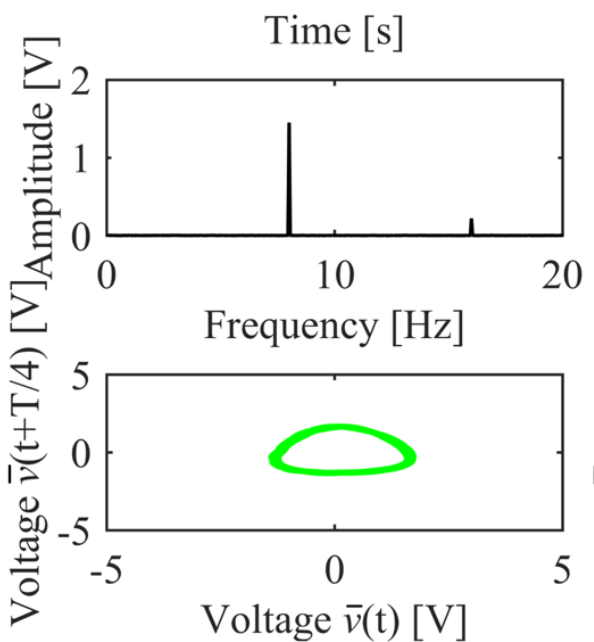

(a)

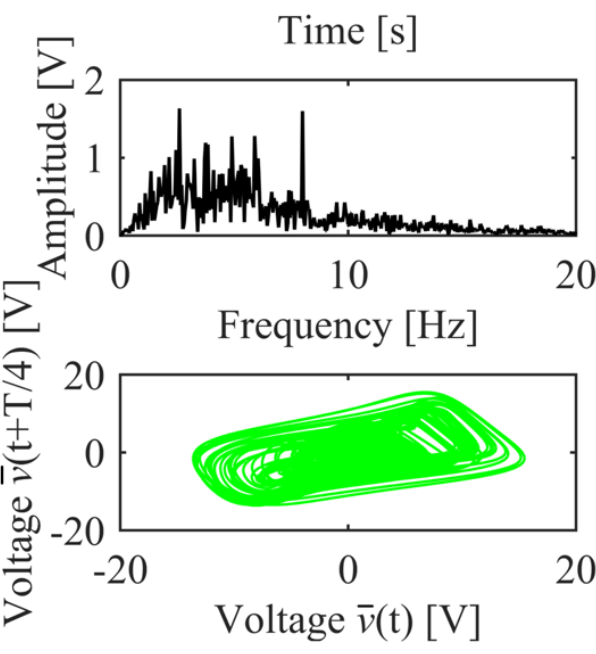

(b)

Fig. 12. Output voltage, frequency spectrum and reconstructed phase portrait of BEH under an excitation of $3 \mathrm{~m} / \mathrm{s}^{2}$ and $8 \mathrm{~Hz}$ with an initial position in the (a) deeper and (b) shallower potential well 

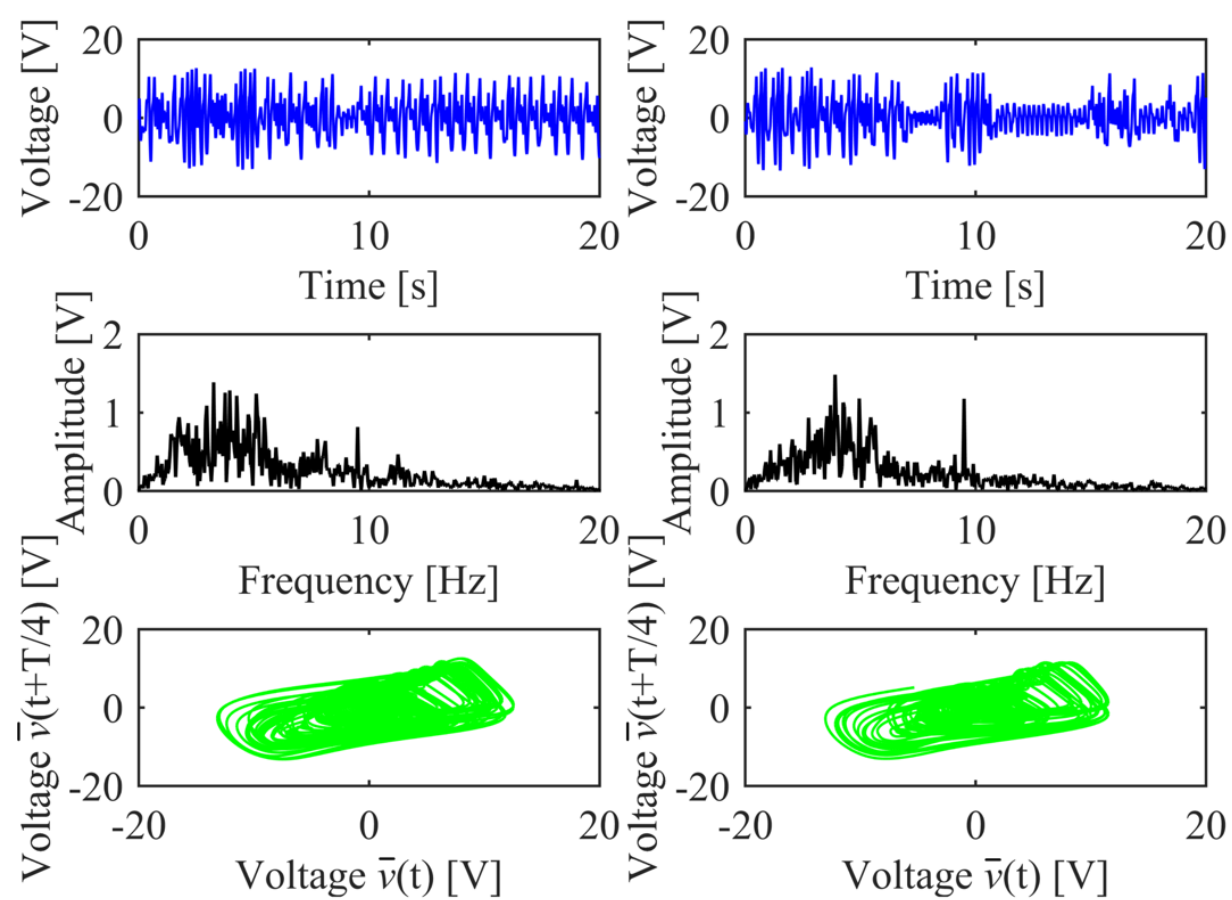

(a)

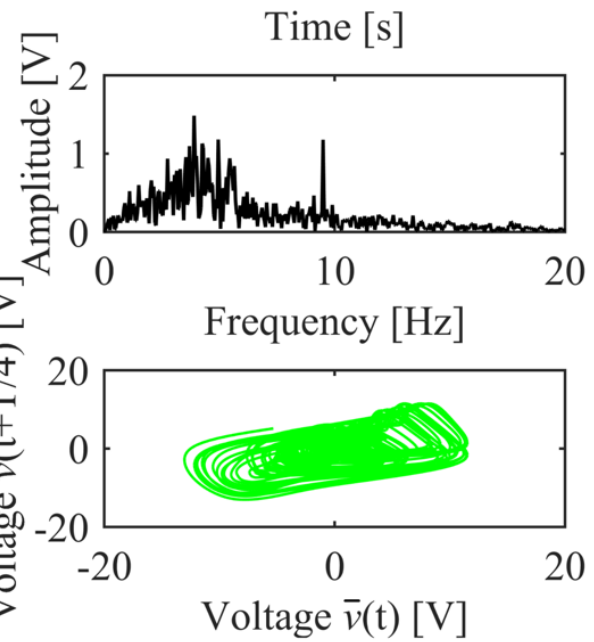

(b)

Fig. 13. Output voltage, frequency spectrum and reconstructed phase portrait of BEH under excitation of $3 \mathrm{~m} / \mathrm{s}^{2}$ and $9.5 \mathrm{~Hz}$ with an initial position in the (a) deeper and (b) shallower potential well
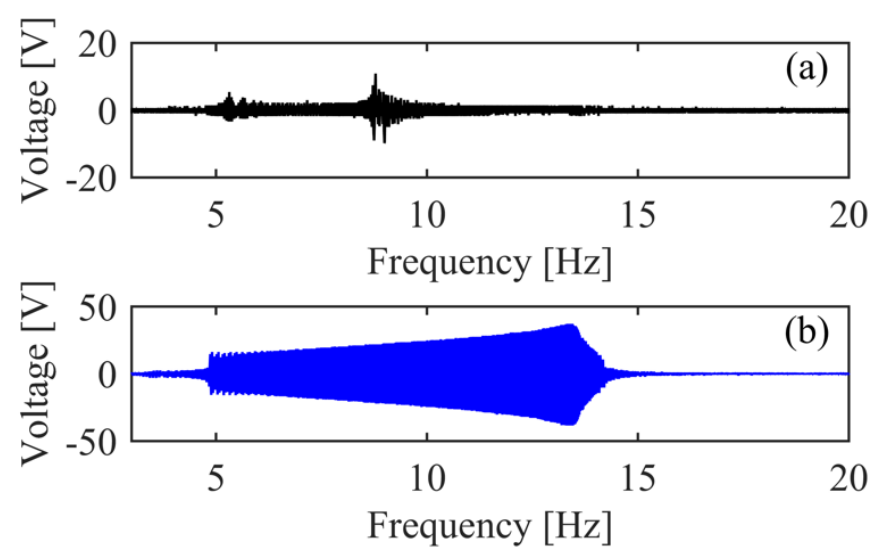

Fig. 14. Voltage response under up-sweep frequency excitation with acceleration of $3 \mathrm{~m} / \mathrm{s}^{2}$ and different initial positions in the (a) deeper and (b) shallower well

In addition, the influence of initial conditions on the response of the asymmetric potential $\mathrm{BEH}$ under sweep frequency excitations is evaluated in the following. Figure 14a and 14b respectively 
show the voltage responses of the asymmetric potential BEH initially oscillating from the deeper and shallower potential wells under up-sweep frequency excitation with acceleration of $3 \mathrm{~m} / \mathrm{s}^{2}$. It is seen from Fig. 14a that there are only several scattered frequencies for the BEH to cross the potential wells and the maximum voltage output is $9 \mathrm{~V}$ at a frequency of $8.75 \mathrm{~Hz}$. However, the bandwidth for inter-well motion of the $\mathrm{BEH}$ is $8.55 \mathrm{~Hz}$ from $4.9 \mathrm{~Hz}$ to $13.45 \mathrm{~Hz}$ when with an initial position in shallower potential well and the maximum output voltage is improved to $37.2 \mathrm{~V}$. Under down-sweep frequency excitation, the output voltages shown in Fig. 15a and 15b are for initially vibrating from the deeper and shallower potential wells, respectively. The BEH firstly oscillates in the deeper potential well and then jumps to the shallower one with the decrease of the frequency, which is the reason why there are two vibration response areas in Fig. 15a. While vibrating from the shallower potential well (see Fig. 15b), the oscillation of the BEH is changing from single well motion in the shallower well to chaotic motion between two wells and then finally to single well motion.

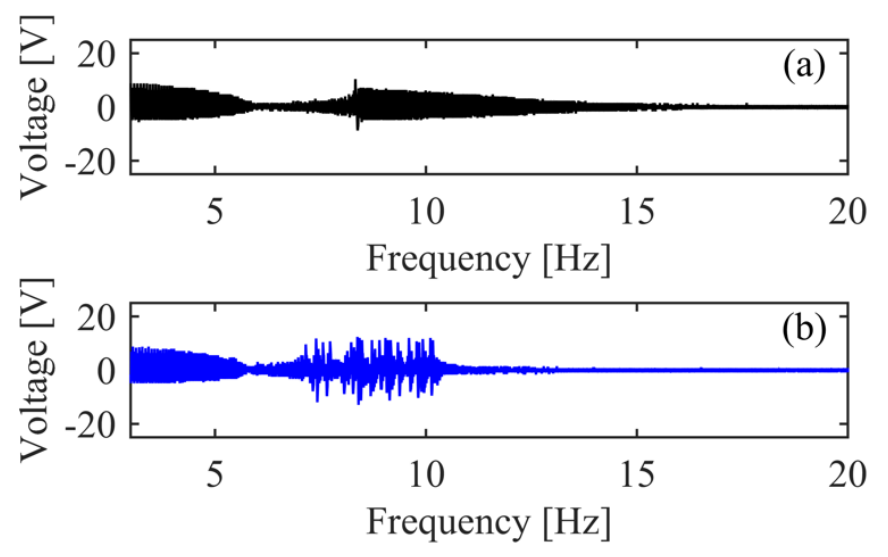

Fig. 15. Voltage response under down-sweep frequency excitation with acceleration of $3 \mathrm{~m} / \mathrm{s}^{2}$ and different initial positions in the (a) deeper and (b) shallower potential well

When the excitation acceleration increases to $4 \mathrm{~m} / \mathrm{s}^{2}$, Figure 16 a illustrates the corresponding voltage response of the BEH with an initial position in deeper potential well under up-sweep frequency excitation. The BEH could travel across the potential well chaotically in the frequency 
range of $9.35 \mathrm{~Hz}$ to $10.08 \mathrm{~Hz}$, which is mainly due to the increase of the excitation acceleration compared to result in Fig. 14a. In contrast, the BEH could achieve inter-well motion on HEB in a wide frequency range from $4.58 \mathrm{~Hz}$ to $13.81 \mathrm{~Hz}$ when initially oscillating from the shallower potential well, thus a maximum voltage of $42.7 \mathrm{~V}$ is achieved. While for down-sweep excitation, see Fig. 17, vibrating from deeper and shallower potential wells all result in chaotic motion of the BEH, but with different effective frequency range. From the experimental results above, one can clearly see the multiple solutions of the asymmetric potential $\mathrm{BEH}$, and conclude that asymmetric potentials influence the voltage output significantly and the performance could be improved not only for constant but also for sweep frequency excitations by initially oscillating from the potential well with shallower depth.

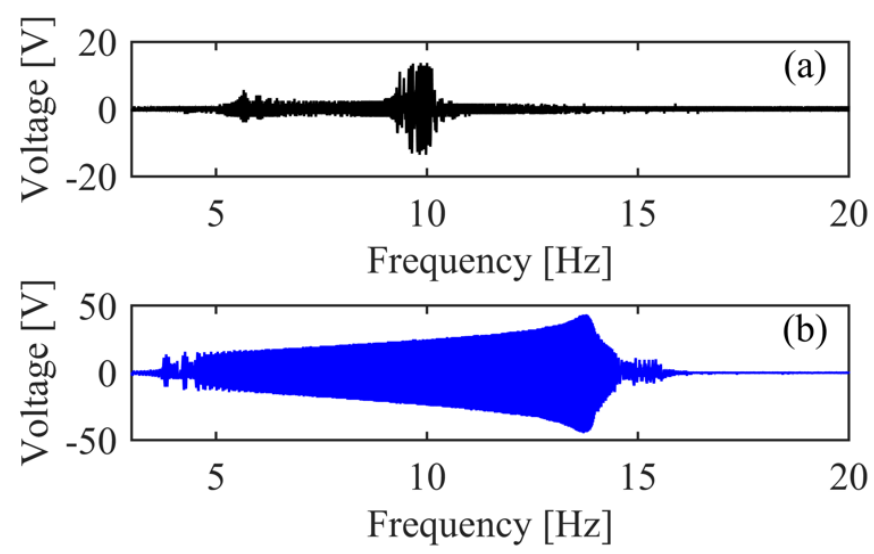

Fig. 16. Voltage response under up-sweep frequency excitation with acceleration of $4 \mathrm{~m} / \mathrm{s}^{2}$ and different initial positions in the (a) deeper and (b) shallower potential well 


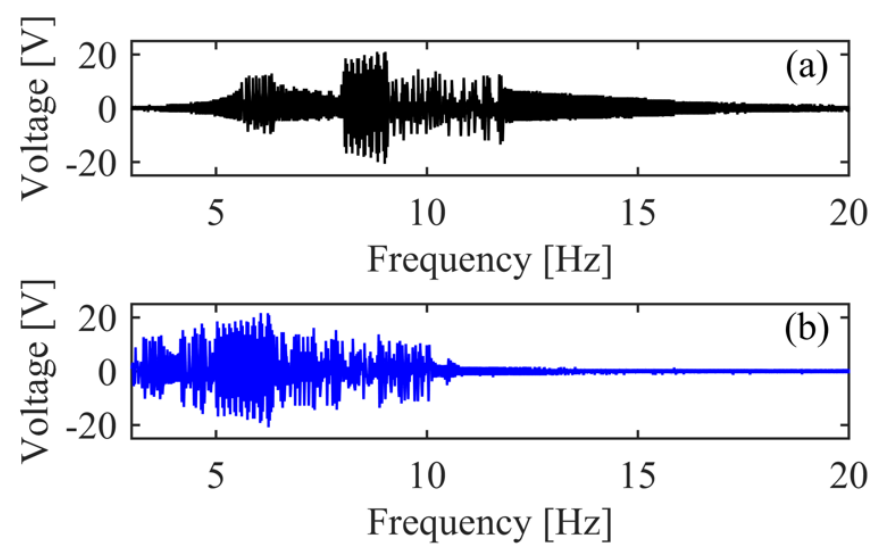

Fig. 17. Voltage response under down-sweep frequency excitation with acceleration of $4 \mathrm{~m} / \mathrm{s}^{2}$ and different initial positions in the (a) deeper and (b) shallower potential well

\section{Conclusion}

The paper presents the detailed numerical and experimental investigations into the multiple solutions of the asymmetric potential bistable energy harvesters (BEHs). Basins of attraction for dimensionless model are firstly applied to illustrate the multiple solutions of the asymmetric potential BEHs due to various initial conditions under different excitation levels, concluding that the appearance of asymmetric potentials in BEHs has mainly a negative effect on the output performance in a relatively small excitation limit. Average output powers of BEHs with different asymmetric degrees under various excitation frequencies and initial conditions demonstrate that there is a higher probability for the asymmetric potential BEHs to achieve high-energy branches (HEBs) and generate a large output power when initially oscillating from the potential well with shallower depth. An asymmetric potential $\mathrm{BEH}$ is modulated to experimentally verify the results from numerical simulation. Experimental data validate the multiple solutions and indicate that the performance of the asymmetric potential BEH can be actually enhanced for not only constant but also sweep frequency excitations by letting the BEH have an initial condition in the shallower potential well. Moreover, we observe an additional solution with even super-harmonic resonance which could contribute to the 
frequency broadband effect for larger excitation levels. The work provides an insight into new strategies to design bistable harvesting system with asymmertric potential wells or account for asymmetries in the potential well of bistable havesting systems due to inherent manufacturing imperfections.

This study was supported by the National Natural Science Foundation of China (Grant No. 51575426, 51611530547, 51811530321), Novel Energy Materials, Engineering Science and Integrated Systems (NEMESIS, Grant No. 320963), and Fundamental Research Funds for the Central Universities of China (Grant No. xjj2016002); this research was partly supported by China Scholarship Council.

\section{Author contribution statement}

All the authors have contributed equally to this work. All the authors were involved in the preparation of the manuscript.

\section{References}

1. P. D. Mitcheson, E. M. Yeatman, G. K. Rao, A. S. Holmes, T. C. Green, Proceedings of the IEEE 96, 1457 (2008).

2. S. P. Beeby, M. J. Tudor, N. M. White, Meas. Sci. Technol. 17, R175 (2006).

3. L. Tang, Y. Yang, C. K. Soh, J. Intell. Mater. Syst. Struct. 21, 1867 (2010).

4. H. A. Sodano, D. J. Inman, G. Park, Shock Vib. Dig. 36, 197 (2004).

5. S. Roundy, P. K. Wright, J. Rabaey, Comput. Commun. 26, 1131 (2003).

6. M. F. Daqaq, J. Sound Vib. 330, 2554 (2011).

7. M. F. Daqaq, R. Masana, A. Erturk, D. Dane Quinn, Appl. Mech. Rev. 66, 040801 (2014).

8. S. Zhou, J. Cao, A. Erturk, J. Lin, Appl. Phys. Lett. 102, 173901 (2013).

9. $\quad$ S. C. Stanton, C. C. McGehee, B. P. Mann, Appl. Phys. Lett. 95, 174103 (2009).

10. Y. Zhang, L. Tang, K. Liu, J. Intell. Mater. Syst. Struct. 28, 307 (2016).

11. K. Fan, Q. Tan, Y. Zhang, S. Liu, M. Cai, Y. Zhu, Appl. Phys. Lett. 112, 123901 (2018).

12. F. Cottone, H. Vocca, L. Gammaitoni, Phys. Rev. Lett. 102, 080601 (2009).

13. A. Erturk, J. Hoffmann, D. J. Inman, Appl. Phys. Lett. 94, 254102 (2009).

14. A. Erturk, D. J. Inman, J. Sound Vib. 330, 2339 (2011).

15. G. Litak, M. I. Friswell, S. Adhikari, Appl. Phys. Lett. 96, 214103 (2010).

16. A. F. Arrieta, T. Delpero, A. E. Bergamini, P. Ermanni, Appl. Phys. Lett. 102, 173904 (2013). 
17. R. Masana, M. F. Daqaq, J. Sound Vib. 332, 6755 (2013).

18. P. Wolszczak, K. Łygas, G. Litak, Mech. Syst. Signal Pr. 107, 43 (2018).

19. P. Kim, J. Seok, J. Sound Vib. 333, 5525 (2014).

20. S. Zhou, J. Cao, D. J. Inman, J. Lin, S. Liu, Z. Wang, Appl. Energ. 133, 33 (2014).

21. J. Cao, S. Zhou, W. Wang, J. Lin, Appl. Phys. Lett. 106, 173903 (2015).

22. H. Li, W. Qin, C. Lan, W. Deng, Z. Zhou, Smart Mat. Struct. 25, 015001 (2016).

23. R. Masana, M. F. Daqaq, J. Appl. Phys. 111, 044501 (2012).

24. J. Cao, S. Zhou, D. J. Inman, Y. Chen, Nonlinear Dyn. 80, 1705 (2015).

25. P. Harris, M. Arafa, G. Litak, C. R. Bowen, J. Iwaniec, Eur. Phys. J. B 90, 20 (2017).

26. P. Harris, C. R. Bowen, H. A. Kim, G. Litak, Eur. Phys. J. Plus 131, 109 (2016).

27. A. Syta, G. Litak, M. I. Friswell, S. Adhikari, Eur. Phys. J. B 89, 99 (2016).

28. A. Syta, G. Litak, M. I. Friswell, M. Borowiec, in Dynamical Systems: Theoretical and Experimental Analysis (Springer, Poland, 2016), pp. 343.

29. R. L. Badzey, P. Mohanty, Nature 437, 995 (2005).

30. J. H. Li, Phys. Rev. E, 66, 031104 (2002).

31. S. Bouzat, H. S. Wio, Phys. Rev. E 59, 5142 (1999).

32. O. Gerashchenko, Tech. Phys. Lett. 29, 256 (2003).

33. Y. Jin, W. Xu, M. Xu, Chaos Soliton Fract. 26, 1183 (2005).

34. S. Jeyakumari, V. Chinnathambi, S. Rajasekar, M. A. F. Sanjuan, Int. J. Bifurcat. Chaos 21, 275 (2011).

35. Q. F. He, M. F. Daqaq, J. Sound Vib. 333, 3479 (2014).

36. E. Halvorsen, Phys. Rev. E 87, 042129 (2013).

37. S. Zhou, L. Zuo, Commun. Nonlinear Sci. 61, 271 (2018).

38. J. Cao, W. Wang, S. Zhou, D. J. Inman, J. Lin, Appl. Phys. Lett. 107, 143904 (2015).

39. W. Wang, J. Cao, C. R. Bowen, D. J. Inman, J. Lin, Appl. Phys. Lett. 112, 213903 (2018).

40. S. Strogatz, in Nonlinear dynamics and chaos: with applications to physics, biology, chemistry, and engineering (Cambridge, MA, 1994).

41. S. Zhou, J. Cao, G. Litak, J. Lin, tm-Tech. Mess. (2018) in press. 